Rev. salud pública. 14 (1): 88-101, 2012

\title{
Perfil gerontológico del adulto mayor en Tabasco, México
}

\section{Gerontological profile of an elderly population in Tabasco, Mexico}

Ramiro Guerrero-Pérez ${ }^{1}$,Elsy del Carmen Quevedo-Tejero² ${ }^{2}$ Ramón García-Rochín ${ }^{3}$ y Marco A. Zavala-González ${ }^{4}$

1 Instituto Nacional de las Personas Adultas Mayores (INAPAM), Delegación Estatal en Tabasco, México. E-mail: rwarrior@prodigy.net.mx

2 División Académica de Ciencias de la Salud (DACS) de la Universidad Juárez Autónoma de Tabasco (UJAT), México. E-mail: dra_elsyquevedo@hotmail.com

3 Secretaría de Salud (SS) del Estado de Tabasco, México. E-mail: ramon.garciar@saludtab.gob.mx 4 "Pensar. Consultores", Sistema Estatal de Investigadores de Tabasco, Sociedad Mexicana de Salud Pública A.C., Asociación Latinoamericana de Profesores de Medicina Familiar A.C.zgma_51083@, yahoo.com.mx

Recibido 10 Abril 2010/Enviado para Modificación 19 Febrero 2012/Aceptado 25 Febrero 2012

\section{RESUMEN}

Objetivo Definir el perfil gerontológico de los adultos mayores en Tabasco, México. Material y métodos Estudio observacional, retrospectivo, transversal, descriptivo. Universo: 35745 adultos mayores afiliados al Instituto Nacional de las Personas Adultas Mayores (INAPAM), Delegación Tabasco. Muestra: no se tomó. Criterios de selección: adultos mayores que se afiliaron al INAPAM durante el período Enero/2004-Diciembre/2006. Variables: socio demográficas, sanitarias y de preferencias personales. Fuente de información: base de datos de la Encuesta Nacional de Adultos Mayores del INAPAM correspondiente al período de estudio. Análisis: estadísticas descriptivas.

Resultados Algunos adultos mayores se encuentran incapacitados para trabajar $(11,9 \%)$, los que laboran lo hacen en el campo (10,5\%). Casi todos tienen ingresos (99,8 \%):por apoyo familiar (46 \%)o empleo (22,3\%). El analfabetismo está en la quinta parte de la población (21,0 \%). La mayoría viven acompañados (88,9\%): por cónyuges (57,3 \%) o hijos (45,9\%). Viven en casas propias $(87,1 \%)$, con los servicios básicos (94,3\%), y cuentan con estufa, televisión y refrigerador (74,5\%). Las enfermedades más frecuentes: osteo degenerativas (50,4\%), oftalmológicas $(39,2 \%)$ y odontológicas (16,3 \%), además de: hipertensión arterial, Diabetes mellitus y neoplasias. Cuentan con seguridad social el 63,3\%, pero los servicios de la Secretaría (Ministerio) de Salud son más usados $(28,7 \%)$. Fomentan los valores: salud (81,2\%), familia (72,4\%), y amor (45,9\%); la convivencia con la familia es la actividad más frecuente de tiempo libre (72\%), junto a las actividades religiosas $(56,4 \%)$

Conclusiones Los rasgos citados integran el perfil gerontológico del adulto mayor 
de Tabasco. Los resultados de esta serie, difieren parcialmente con los reportados por otras instituciones nacionales.

Palabras Clave: Anciano, encuestas epidemiológicas, encuesta socioeconómica, perfil de salud (fuente: DeCS, BIREME).

\section{ABSTRACT}

Objective Defining the gerontological profile of an elderly population in Tabasco, Mexico.

Material and methods This was an observational, retrospective, cross-sectional, descriptive study. The study population consisted of 35,745 elderly adults who were enrolled in the Mexican Older Adults' Institute (Instituto Nacional de las Personas Adultas Mayores-INAPAM) in Tabasco. Sampling was not used. The selection criteria involved elderly adults who were enrolled in INAPAM from January/2004December/2006. Socio-demographic, sanitary and personal preferences were the variables considered. INAPAM's Mexican Survey of Senior Citizens' database for the study period was used for providing the necessary information; descriptive statistics were used for analysis.

Results Some elderly adults were unable to work (11.9\%); those working did so in the countryside $(10.5 \%)$. Almost all of them had some sort of income (99.8 $\%)$ : family support (46\%) or employment $(22.3 \%)$. A fifth of the population were illiterate $(21 \%)$. Most of them lived with someone else (88.9\%) with a spouse $(57.3$ $\%$ ) or children (45.9\%). Most lived in their own houses (87.1\%), had basic services $(94.3 \%)$ and a stove, television and refrigerator (74.5\%). The frequently occurring diseases were osteo-degenerative $(50.4 \%)$, ophthalmic $(39.2 \%)$ dental (16.3 $\%)$, high blood pressure-related, Diabetes mellitus and tumours. Most had social security (63.3 \%) but mostly used Mexican Ministry of Health services (28.7 \%). They fostered the following values: health (81.2\%), family (72.4\%) and love (45.9 $\%)$; sharing family time was the most frequent free-time activity $(72 \%)$ followed by religious activities (56.4\%).

Conclusions The aforementioned features formed the gerontological profile for elderly adults in Tabasco; and the results of this series partially differed from that reported by other Mexican institutions.

Key Words: Aged, health survey, socioeconomic survey, health profile (source: $\mathrm{MeSH}, \mathrm{NLM})$.

$\mathrm{L}$ a transición demográfica, es la base para la construcción de políticas públicas, en pro de la investigación sobre el envejecimiento de la población (1). El estudio de la población adulta mayor, cobra importancia debido a que se traduce en personas viviendo más años provocando cambios socio demográficos, sanitarios y de preferencias personales (1). Tabasco, actualmente con una población joven en relación con otros Estados de la República Mexicana, no está exento del envejecimiento 
demográfico, por lo que conocer el perfil gerontológico de esta población, permitiría planear mejor estrategias y políticas apropiadas para hacerle frente.

En Tabasco, México, las investigaciones tendientes a establecer un perfil del adulto mayor son dispersas y escasas. La información disponible actualmente, proviene de organismos nacionales como el Instituto Nacional de Estadística, Geografía e Informática (INEGI) (2-4) y el Consejo Nacional de Población (CONAPO) (5) cuyos reportes se enfocan en aspectos demográficos; se cuenta también con información proveniente del Estudio Nacional de Salud y Envejecimiento en México (ENASEM) (6) y la Encuesta Salud, Bienestar y Envejecimiento (SABE) (7), que ofrecen una descripción más amplia, con carencias en rubros como los bienes.

En este contexto, surge la Encuesta de Adultos Mayores (EAM), aplicada por el Instituto Nacional para las Personas Adultas Mayores (INAPAM), que integra variables socioeconómicas, sanitarias y de preferencias personales, consideras importantes por mostrar específicamente las características del adulto mayor en Tabasco. Ésta, cuenta con deficiencias estructurales y procesales comentadas por Ham-Chande y cols.(8). No obstante, contiene datos útiles, por lo que se realizó un estudio con el objetivo de caracterizar el perfil gerontológico de las personas adultas mayores en el Estado de Tabasco, México, con base en la EAM del INAPAM.

\section{MATERIAL Y MÉTODOS}

Se realizó un estudio observacional, retrospectivo, transversal y descriptivo, en el año 2008. La información provino de la base de datos de la EAM aplicada por el INAPAM al adulto mayor en el momento de solicitar su credencial de afiliación durante el periodo 2004-2006, en la Delegación Estatal de Tabasco. El universo de estudio estuvo constituido por $\mathrm{N}=35745$ adultos mayores que obtuvieron su afiliación durante el periodo citado. No se tomó muestra, se estudió al universo en su totalidad.

Las variables consideradas en este estudio se agruparon en: 1 . Socio demográficas: situación laboral, ocupación, temporalidad del trabajo, fuente de ingresos, ingreso promedio mensual, alfabetismo, con quien vive, es jefe del hogar, activos fijos en el hogar, posesión de la vivienda, servicios en la vivienda, combustible empleado para cocinar, y material del piso de la vivienda; 2. Sanitarias: padecimientos más frecuentes, institución de derecho habiencia, derecho habiencia por familiares, y servicios médicos utilizados; 
3. Preferencias Personales: valores, actividades de tiempo libre y ocio, actividades que gustaría de realizar en tiempo libre.

La base de datos contaba con números crudos, no editables. El error más significativo fue la falta de especificación de respuestas múltiples, ya que la EAM contiene preguntas con posibilidad de más de una respuesta. Dadas las características de la base de datos, no se pudo determinar cuáles registros pertenecían a una misma persona en las preguntas de respuestas múltiples. Por esta estructura de la base de datos, no se tuvo la posibilidad de realizar un análisis cruzado de las variables luego de su decodificación.

La base de datos (previamente decodificada) fue sometida a análisis en tres etapas. Primera etapa: los datos contenidos en el banco electrónico fueron sometidos a un proceso de depuración, y de selección de las variables previamente especificadas; en primer término, en los datos proporcionados por el INAPAM se encontraron excluidas las variables correspondientes a la ficha de identificación (edad, sexo, procedencia y lugar de nacimiento); en segundo lugar, se eliminaron los registros ambiguos cuyos valores no aportan información de interés para este estudio. Segunda etapa: como resultado de la depuración de datos inservibles se estableció un diagnóstico inicial, con la finalidad de reconocer las posibles limitaciones y sesgos de la información. Tercera etapa: se obtuvieron estadísticas descriptivas para los datos socio demográficos, sanitarios y de preferencias personales rescatados; con base en éstos, datos se integró el "perfil gerontológico" de la población de adultos mayores de Tabasco. Los datos utilizados y los cálculos realizados, fueron tabulados y analizados empleando el software Microsoft ${ }^{\circledR}$ Excel ${ }^{\circledR}$ versión 2003 para entorno Windows ${ }^{\circledR}$.

\section{RESULTADOS}

Se enuncian por secciones, los principales resultados obtenidos del análisis de la EAM, finalizando con la integración de la información en el "perfil gerontológico" del adulto mayor de Tabasco, México.

Aspectos socio demográficos

Los resultados de las variables: situación laboral, ocupación, fuente de ingresos, con quiénes viven, activos fijos en el hogar y servicios en la vivienda; se refieren a las proporciones individuales en que fue respondida cada opción. Mientras que los resultados de las variables: temporalidad del trabajo, ingreso promedio mensual, alfabetismo, jefes del hogar, posesión 
de la vivienda, combustible empleado para cocinar y material del piso de la vivienda; corresponden a las distribución de frecuencias de la variable, suman $100 \%$ en cada una. Los resultados se muestran en la Tabla 1.

Tabla 1. Aspectos socio demográficos ( $n=35745)$

\begin{tabular}{|c|c|c|c|}
\hline & Variable & Frecuencia & $\%$ \\
\hline \multirow{5}{*}{$\begin{array}{l}\text { Situación } \\
\text { laboral }\end{array}$} & Dedicado al hogar & 1374 & 3,8 \\
\hline & Está incapacitado para trabajar & 4241 & 11,9 \\
\hline & Está jubilado & 10299 & 28,8 \\
\hline & Pensionado & 13906 & 38,9 \\
\hline & Trabaja & 23203 & 64,9 \\
\hline \multirow{12}{*}{ Ocupación } & Miembro de una cooperativa & 50 & 0,1 \\
\hline & Patrón o empleador de un negocio & 159 & 0,4 \\
\hline & Actividad familiar con retribución & 199 & 0,6 \\
\hline & Profesionista independiente & 212 & 0,6 \\
\hline & Actividad familiar sin retribución & 322 & 0,9 \\
\hline & Albañil & 379 & 1,1 \\
\hline & Empleado(a) doméstico(a) & 389 & 1,1 \\
\hline & Comerciante & 628 & 1,8 \\
\hline & Obrero & 842 & 2,4 \\
\hline & Trabajador por cuenta propia & 1719 & 4,8 \\
\hline & Empleado & 2302 & 6,4 \\
\hline & Ejidatario o peón de campo & 3761 & 1,5 \\
\hline \multirow{3}{*}{$\begin{array}{l}\text { Temporalidad } \\
\text { del trabajo }\end{array}$} & No contestaron* & 25216 & 70,5 \\
\hline & Temporal & 4516 & 12,6 \\
\hline & Permanente & 6013 & 16,8 \\
\hline \multirow{9}{*}{$\begin{array}{l}\text { Fuente de } \\
\text { ingresos }\end{array}$} & Renta, inversión o ahorro & 123 & 0,3 \\
\hline & Cónyuge & 430 & 1,2 \\
\hline & Negocio & 1118 & 3,1 \\
\hline & No contestaron* & 3096 & 8,7 \\
\hline & Otro & 3149 & 8,8 \\
\hline & Jubilación y pensión & 4594 & 12,9 \\
\hline & Empleo & 7958 & 22,3 \\
\hline & Ayuda de familiares & 16451 & 46,0 \\
\hline & No tienen ingresos & 68 & 0,2 \\
\hline \multirow{8}{*}{$\begin{array}{l}\text { Ingreso pro- } \\
\text { medio men- } \\
\text { sual }\end{array}$} & No contesto* & 2011 & 5,6 \\
\hline & Menos de $\$ 1000$ & 14877 & 41,6 \\
\hline & $\$ 1001$ a $\$ 2000$ & 3983 & 11,1 \\
\hline & $\$ 2001$ a $\$ 3000$ & 1369 & 3,8 \\
\hline & $\$ 3001$ a $\$ 4000$ & 866 & 2,4 \\
\hline & $\$ 4001$ a $\$ 5000$ & 673 & 1,9 \\
\hline & Más de $\$ 5001$ & 1384 & 3,9 \\
\hline & No sabe & 10582 & 29,6 \\
\hline
\end{tabular}




\begin{tabular}{|c|c|c|c|}
\hline & Variable & Frecuencia & $\%$ \\
\hline \multirow{3}{*}{$\begin{array}{l}\text { Saben leer y } \\
\text { escribir }\end{array}$} & No contestaron* & 1022 & 2,9 \\
\hline & $\mathrm{Si}$ & 27208 & 76,1 \\
\hline & No & 7515 & 21,0 \\
\hline \multirow{7}{*}{$\begin{array}{l}\text { Con quienes } \\
\text { viven }\end{array}$} & Padres & 468 & 1,3 \\
\hline & Otros no familiares & 954 & 2,7 \\
\hline & Otros familiares & 2220 & 6,2 \\
\hline & Viven solos & 3952 & 11,1 \\
\hline & Nietos & 5784 & 16,2 \\
\hline & Hijos & 16418 & 45,9 \\
\hline & Cónyuge & 20493 & 57,3 \\
\hline \multirow{3}{*}{$\begin{array}{l}\text { Jefes del } \\
\text { hogar }\end{array}$} & No contestaron* & 1082 & 3,0 \\
\hline & $\mathrm{Si}$ & 27343 & 76,5 \\
\hline & No & 7320 & 20,5 \\
\hline \multirow{10}{*}{$\begin{array}{l}\text { Artículos en el } \\
\text { hogar }\end{array}$} & Computadora & 1815 & 5,1 \\
\hline & Teléfono celular & 2674 & 7,5 \\
\hline & Automóvil & 2708 & 7,6 \\
\hline & Video casetera & 2999 & 8,4 \\
\hline & Teléfono fijo & 10226 & 28,6 \\
\hline & Lavadora & 13307 & 37,2 \\
\hline & Radio & 20251 & 56,7 \\
\hline & Refrigerador & 25368 & 71,0 \\
\hline & Televisión & 26015 & 72,8 \\
\hline & Estufa de gas & 28534 & 79,8 \\
\hline \multirow{6}{*}{$\begin{array}{l}\text { Propiedad de } \\
\text { la vivienda }\end{array}$} & No contestaron* & 1054 & 2,9 \\
\hline & Propia & 31132 & 87,1 \\
\hline & Pagan renta & 915 & 2,6 \\
\hline & Otra persona les paga la renta & 340 & 1,0 \\
\hline & Prestada & 2246 & 6,3 \\
\hline & Albergue o asilo & 58 & 0,2 \\
\hline \multirow{3}{*}{$\begin{array}{l}\text { Servicios en } \\
\text { la vivienda }\end{array}$} & Agua corriente & 33171 & 92,8 \\
\hline & Luz eléctrica & 33484 & 93,7 \\
\hline & Baño o letrina & 34463 & 96,4 \\
\hline \multirow{5}{*}{$\begin{array}{l}\text { Combustible } \\
\text { empleado } \\
\text { para cocinar }\end{array}$} & No contestaron* & 1375 & 3,8 \\
\hline & Electricidad & 131 & 0,4 \\
\hline & Petróleo & 107 & 0,3 \\
\hline & Leña o carbón & 4781 & 13,4 \\
\hline & Gas & 29351 & 82,1 \\
\hline \multirow{6}{*}{$\begin{array}{l}\text { Material del } \\
\text { piso de la } \\
\text { vivienda }\end{array}$} & No contestaron* & 1036 & 2,9 \\
\hline & Tierra & 1443 & 4,0 \\
\hline & Cemento & 28068 & 78,5 \\
\hline & Mosaico & 5036 & 14,1 \\
\hline & Madera & 118 & 0,3 \\
\hline & Ninguno & 44 & 0,1 \\
\hline
\end{tabular}


Tabla 2. Aspectos sanitarios ( $n=35$ 745)

\begin{tabular}{|c|c|c|c|}
\hline & Variable & Frecuencia & $\%$ \\
\hline \multirow{16}{*}{$\begin{array}{l}\text { Aspectos } \\
\text { sanitarios }\end{array}$} & Cáncer & 370 & 1,0 \\
\hline & Próstata o cervicouterino & 1849 & 5,2 \\
\hline & Osteoporosis & 2136 & 6,0 \\
\hline & Respiratorios & 2636 & 7,4 \\
\hline & Depresión & 2713 & 7,6 \\
\hline & Columna vertebral & 3015 & 8,4 \\
\hline & Artritis & 3506 & 9,8 \\
\hline & Corazón & 3712 & 10,4 \\
\hline & Gástricos & 3751 & 10,5 \\
\hline & Circulatorios & 4818 & 13,5 \\
\hline & Ninguna & 5749 & 16,1 \\
\hline & Dentadura & 5809 & 16,3 \\
\hline & Diabetes & 5859 & 16,4 \\
\hline & Reumáticos & 9368 & 26,2 \\
\hline & Hipertensión arterial & 10333 & 28,9 \\
\hline & Enfermedades de la vista & 13995 & 39,2 \\
\hline \multirow{6}{*}{$\begin{array}{l}\text { Institución } \\
\text { de Derecho } \\
\text { habiencia }\end{array}$} & IMSS & 18232 & 51,0 \\
\hline & ISSSTE & 4742 & 13,3 \\
\hline & Marina o Defensa & 2325 & 6,5 \\
\hline & Ninguno & 3343 & 9,4 \\
\hline & No contestaron & 6968 & 19,5 \\
\hline & PEMEX & 135 & 0,4 \\
\hline \multirow{6}{*}{$\begin{array}{l}\text { Derecho } \\
\text { habiencia de } \\
\text { familiares }\end{array}$} & IMSS & 6158 & 17,2 \\
\hline & ISSSTE & 3108 & 8,7 \\
\hline & Marina o Defensa & 136 & 0,4 \\
\hline & Ninguno & 5848 & 16,4 \\
\hline & No contestaron & 18311 & 51,2 \\
\hline & PEMEX & 2184 & 6,1 \\
\hline \multirow{11}{*}{$\begin{array}{l}\text { Servicios } \\
\text { médicos } \\
\text { utilizados }\end{array}$} & Clínica u hospital privado & 2547 & 7,1 \\
\hline & Dispensarios & 504 & 1,4 \\
\hline & Farmacias & 221 & 0,6 \\
\hline & IMSS & 5968 & 16,7 \\
\hline & INAPAM & 135 & 0,4 \\
\hline & ISSSTE & 3057 & 8,6 \\
\hline & Ninguno & 2077 & 5,8 \\
\hline & No contestaron & 1041 & 2,9 \\
\hline & Otros servicios públicos & 4724 & 13,2 \\
\hline & Secretaría de Salud & 10243 & 28,7 \\
\hline & Seguro Popular & 5228 & 14,6 \\
\hline
\end{tabular}

Aspectos sanitarios

Los resultados de la variable "padecimientos más frecuentes", se refieren a las 
proporciones individuales en que fue respondida cada opción. Mientras que los resultados de las variables: institución de derecho habiencia, derecho habiencia por familiares y servicios médicos utilizados; corresponden a las distribución de frecuencias de la variable, suman $100 \%$ en cada una. Los resultados se muestran en la Tabla 2.

Tabla 3. Preferencias personales ( $n=35$ 745)

\begin{tabular}{|c|c|c|c|}
\hline & Variable & Frecuencia & $\%$ \\
\hline \multirow{8}{*}{ Valores } & Religiosos/Espirituales & 5274 & 14,8 \\
\hline & Dinero & 5791 & 16,2 \\
\hline & No depender de otros & 6432 & 18,0 \\
\hline & No sentirse solo & 7262 & 20,3 \\
\hline & Sentirse útil & 7984 & 22,3 \\
\hline & Amor & 16424 & 45,9 \\
\hline & Familia & 25863 & 72,4 \\
\hline & Salud & 29010 & 81,2 \\
\hline \multirow{12}{*}{$\begin{array}{l}\text { Actividades de } \\
\text { tiempo libre }\end{array}$} & Ir al cine/teatro & 671 & 1,9 \\
\hline & Actividades manuales & 1475 & 4,1 \\
\hline & Ir a clubes de la 3ra edad & 2075 & 5,8 \\
\hline & Deporte/hacer ejercicio & 3388 & 9,5 \\
\hline & Relacionarse con amigos & 5203 & 14,6 \\
\hline & Viajar o ir de paseo & 5675 & 15,9 \\
\hline & Oír radio & 6690 & 18,7 \\
\hline & Ver televisión & 8149 & 22,8 \\
\hline & Leer & 12010 & 33,6 \\
\hline & Descansar & 12395 & 34,7 \\
\hline & Actividades religiosas & 20146 & 56,4 \\
\hline & Convivir con la familia & 25741 & 72,0 \\
\hline \multirow{13}{*}{$\begin{array}{l}\text { Actividades que } \\
\text { les gustarían } \\
\text { realizar }\end{array}$} & Ir al cine/teatro & 1862 & 5,2 \\
\hline & Leer & 3817 & 10,7 \\
\hline & Deporte/Hacer ejercicio & 3913 & 10,9 \\
\hline & Ver televisión & 4475 & 12,5 \\
\hline & Tener empleo & 4545 & 12,7 \\
\hline & Oír radio & 5008 & 14,0 \\
\hline & Ir a clubes de la 3ra edad & 7215 & 20,2 \\
\hline & Viajar o ir de paseo & 7469 & 20,9 \\
\hline & Descansar & 11934 & 33,4 \\
\hline & Actividades religiosas & 12151 & 34,0 \\
\hline & Aprender algo nuevo & 12534 & 35,1 \\
\hline & Convivir con otras personas & 12662 & 35,4 \\
\hline & Convivir con la familia & 16377 & 45,8 \\
\hline
\end{tabular}


Preferencias personales

Los resultados de las tres variables que componen el grupo de preferencias personales se refieren a las proporciones individuales en que fue respondida cada opción. Los resultados se muestran en la Tabla 3.

"Perfil gerontológico" del adulto mayor en el Estado de Tabasco, México. Con base en el conjunto de rasgos socio demográficos, sanitarios y de preferencias personales enunciados en los párrafos precedentes, se integró el "perfil gerontológico" del adulto mayor de Tabasco:

La mitad de los adultos mayores presentan alguna discapacidad que les impide laborar, los que si están en condición para ello, lo hacen en su mayoría en el campo, y los menos como empleados generales. La mitad cuenta con ingresos económicos provenientes de la familia, en menor proporción de remuneración laboral y los menos por jubilación o pensión. El promedio mensual de ingresos que perciben es de menos de $\$ 1000,00$ pesos mexicanos. Tres cuarta partes saben leer y escribir. Viven integrados en forma nuclear, en su mayoría siendo ellos jefes de familia y propietarios de la vivienda, la cual cuenta con pisos de cemento, y los servicios básicos. Poseen activos fijos en el hogar que les proporcionan confort y comodidad. Además de la hipertensión arterial, Diabetes mellitus y neoplasias, son afectados por padecimientos osteodegenerativos, oftalmológicos y odontológicos. Los que cuentan con seguridad social están afiliados mayormente al IMSS, sin embargo, la mayoría recurren a los servicios médicos de la Secretaria de Salud. Dentro de sus valores prioritarios están: salud, familia y amor. Ocupan su tiempo libre en la convivencia familiar, actividades religiosas, el descanso y la lectura. Tienen como aspiración prioritaria la convivencia familiar, así como aprender cosas nuevas y la participación en actividades religiosas o espirituales.

\section{DISCUSIÓN}

Dada la carencia de estudios que integren la información concerniente a lo que hemos denominado "perfil gerontológico" (aludiendo a la visión integral del envejecimiento y sus problemáticas), fue necesario el contraste aislado de los diversos rubros de la EAM contra los reportes prexistentes, cuando ello fue posible. Se exponen a continuación las divergencias observadas.

Aspectos socio demográficos A nivel nacional, una significativa proporción de los adultos mayores según 
el INEGI $(2,4)$, permanecen en actividad económica, 36,5 \% de 9 millones de adultos mayores. En Tabasco el 64,9 \% se encuentran económicamente activos (2,3). Esta diferencia, pudiera basarse en la posición que Tabasco ocupa en México de acuerdo a su Producto Interno Bruto (PIB) per capita (4).

El adulto mayor tabasqueño económicamente activo desempeña "labores del campo" en 10,5\%, en tanto que a nivel nacional es $35,1 \%(2,4)$; en funciones de empleado general un $6,4 \%$ en el orden estatal y en el nacional $32,3 \%(2,4)$, y de manera "independiente o cuenta propia" $4,8 \%$ en Tabasco y $27,3 \%$ a nivel nacional $(2,4)$. Estos porcentajes demuestran amplia diferencia en lo referente a las actividades agropecuarias y como empleado general; mientras que en lo que respecta a los empleos de "manera independiente" el índice nacional se encuentra por arriba del estatal $(2,4)$.

Los adultos mayores en Tabasco perciben sus ingresos económicos en $44,5 \%$ del apoyo familiar, en tanto que al nivel nacional, es el 33,7 \% (6); por su empleo a nivel estatal un 21,5\%, mientras que en el nacional es de 41,9\% (6); los ingresos por jubilación o pensión del adulto mayor tabasqueño es del 12,4 \% y nacional de 25,1 \% (6). Las condiciones económicas del Estado, influyen en un mayor apoyo económico familiar.

El INEGI $(2,3)$ reporta que el 31,4 \% de los adultos mayores en México reciben menos de $\$ 1000$ pesos mexicanos al mes, el 23,7\% de $\$ 1001$ a \$2 000 y más de \$2 001 el $7 \%$. Mientras que en la EAM, se observó que poco menos de la mitad $(41,6 \%)$ perciben mensualmente en promedio $\$ 1$ 000 pesos mexicanos, el $11,1 \% \$ 1001$ - $\$ 2000$ y el $6 \%$ con más de $\$ 2$ 001. Los ingresos en Tabasco son menores que el promedio nacional. Estas características económicas de la población, son compatibles con un nivel socioeconómico medio-bajo a bajo, lo cual, según las teorías expuestas por Moragas (10), imbuye de limitaciones a la población.

Por otra parte, la EAM reporta $21 \%$ de analfabetismo, mientras que el INEGI reporta $16,4 \%$ en esta misma entidad (4), en tanto que a nivel nacional, de acuerdo a la proyección del CONAPO (5) lo es 30,1\%. Esto podría relacionarse con la obligatoriedad de que los entonces niños asistieran a la "Escuela Rural Nacionalista" durante el gobierno de Tomas Garrido Canabal (1919, 1923 y 1931) (11). 
En lo que respecta a con quien viven los adultos mayores, de acuerdo al INEGI (3), la mayoría viven con sus familiares, en el $25 \%$ de los hogares hay al menos un adulto mayor, y en el $5 \%$ viven solos. La EAM, por su parte, indica que el 11,1\% viven solos, o con familiares siguiendo esta distribución: 57,3 \% con cónyuge, 45,9\% con hijos(as), 16,2 \% con nietos (as) y $6,2 \%$ con otros. Estos datos no tienen comparativo con los reportados por el INEGI (3).

Según el INEGI (4), en 2005, en Tabasco, 71,3 \% de los adultos mayores contaban con los 3 servicios básicos, en tanto que a nivel nacional eran 79,8\%. Mientras que por su parte, la EAM señala que el 94,3\% de los adultos mayores en Tabasco cuentan con dichos servicios.

Con respecto a la posesión de la vivienda la EAM indica que 87,1 \% de los adultos mayores tabasqueños viven en casa propia, ubicando a Tabasco por arriba de la media nacional de $79 \%$ (3).

Se observó que el combustible empleado para cocinar generalmente fue gas $(82,1 \%)$, y que $13,4 \%$ usan leña o carbón, mientras que otros combustibles son usados en menos de $5 \%$. Tales resultados, difieren con los indicadores nacionales: $76 \%$ gas, $22 \%$ leña, $0,3 \%$ carbón y $0,2 \%$ electricidad (4).

En lo referente a los pisos de las viviendas, los materiales distintos a tierra prevalecen en $89 \%$ a nivel nacional y en 90,6 \% en Tabasco según el INEGI (4). En el presente estudio la proporción fue $92,6 \%$, diferencia mínima, quizá influenciada por las políticas estatales y federales aplicadas.

Aspectos sanitarios

En esta serie, la población refiere padecimientos osteodegenerativos, oftalmológicos y odontológicos, con mayor frecuencia que los índices oficiales reportados por la Secretaría de Salud para el mismo período (9), pero coinciden en la prevalencia de hipertensión arterial, tumores malignos y Diabetes mellitus; lo que podría señalar una subestimación los primeros por el organismo aludido.

Según el INEGI (2), en 2004, el 75,4\% de los derechohabientes del IMSS eran de 60 años y más, en el ISSSTE 17,6 \%, y en otras instituciones públicas como PEMEX, Secretaría de la Defensa Nacional, Secretaría de Marina y Seguro Popular de la Secretaría de Salud 5,5\%. Mientras que los presentes resultados, señalan que en Tabasco $51 \%$ son derechohabientes del 
IMSS, 13,3 \% del ISSSTE, y menos de $10 \%$ de otras instituciones. En todas las instituciones la proporción fue menor a la media nacional, lo que puede atribuirse a que la planta productiva del Estado es de jóvenes (5), o a que los adultos mayores cuentan con oportunidades de empleo restringidas (3).

Preferencias personales

Los valores más arraigados en los adultos mayores de Tabasco observados fueron: salud $81,2 \%$, familia $74,4 \%$, y amor $45,9 \%$; para los que no se encontró precedente. Asimismo, refirieron usar su tiempo libre en $72 \%$ para convivir con la familia, 56,4 \% actividades religiosas, 34,7 \% descansar y $33,6 \%$ leer. Tales resultados difieren con lo reportado por $\mathrm{Su} B$ y cols. (12), Alcocea y cols. (13), Martín-Sánchez y cols. (14), y Sánchez-García y cols. (15), quienes reportan que ver televisión es la actividad más frecuente, y que el cuidado de los nietos (como convivencia familiar) ocupa un lugar muy lejano dentro de las actividades que realizan los adultos mayores. Las actividades de los adultos mayores de Tabasco, reflejan integración familiar y desarrollo espiritual.

Finalmente, las principales aspiraciones del adulto mayor resultaron ser: convivir con la familia y/o con otras personas y aprender cosas nuevas; tales aspiraciones denotan la predilección por llevar a cabo actividades de integración familiar, y demuestran necesidad de integración social, así como de realizar actividades que les permitan sino desarrollarse intelectualmente, si preservar sus capacidades mentales.

Los resultados obtenidos permiten identificar que los adultos mayores en Tabasco, México, se desempeñan laboralmente en el campo lo que hace necesario que los servicios y políticas sociales se enfoquen a este ámbito, mejorando la atención socio-sanitaria y de desarrollo personal.

Es necesario mejorar la estructura y procedimiento de sistematización de la EAM, incluyendo variables que permitan conocer con mayor profundidad la problemática del envejecimiento para futuros estudios, como la exploración de condiciones concretas como demencia, deterioro cognitivo, abandono, maltrato y polifarmacia, entre otros síndromes geriátricos en posibilidad de explorarse mediante la aplicación de este instrumento, y por medio del empleo de otros entornos operativos y sistemas de bases de datos que permitan la captura de variables con múltiples respuestas y análisis cruzado de las mismas 


\section{REFERENCIAS}

1. Secretaría de las Naciones Unidas. Revisiones demográficas mundiales. Revisión 2006. Nueva York, E.U.A.: ONU; 2007.

2. Instituto Nacional de Estadística, Geografía e Informática (INEGI). Encuesta Nacional de Empleo y Seguridad Social 2004 (ENESS-2004) [Internet]. México: INEGI, 2005. Disponible en http://www.google.com.mx/url?sa=t\&source=web\&cd=1\&ved=0CBIQ FjAA\&url=http\%3A\%2F\%2Fwww.inegi.gob.mx\%2Fprod serv\%2Fcontenidos\%2Fe spanol\%2Fbiblioteca\%2Fabrepdf.asp\%3Fupc\%3D702825470388\&ei=RLKeTfH6C oPa0QGBorjbBw\&usg=AFQjCNHSbshHcCPNT2LU_rJrEgVqpbGbBg Consultado: abril 2011.

3. Instituto Nacional de Estadística, Geografía e Informática (INEGI). Los adultos mayores en México. Perfil sociodemográfico al Inicio del Siglo XXI [Internet]. México: INEGI, 2005. Disponible en http://www.google.com.mx/url?sa=t\&source=web\&cd=1\&ved= OCBIQFjAA\&url=http\%3A\%2F\%2Fwww.inegi.gob.mx\%2Fprod_serv\%2Fcontenido s\%2Fespanol\%2Fbiblioteca\%2Fabrepdf.pc\%3D702825001083\&ei=D7OeTbAcg4z RAcLCoYoF\&usg=AFQjCNGBuU9kknn68jyoDA7TkD7UBBsFHg Consultado: abril 2011.

4. Instituto Nacional de Estadística, Geografía e Informática (INEGI). Resultados definitivos del II Censo Nacional de Población y Vivienda 2005 para el Estado de Tabasco [Internet]. México: INEGI, 2006 Disponible en http://www.google.com.mx/url?sa=t\&source=web \&cd=3\&ved=0CCEQFjAC\&url=http\%3A\%2F\%2Fwww.inegi.org.mx\%2Finegi\%2Fco ntenidos\%2Fespanol\%2Fprensa\%2FBoletines\%2FBoletin\%2FComunicados\%2FEs peciales\%2F2006\%2FMayo\%2Fcomunica11.f\&ei=SLGeTe7bHqSO0QGemcikBQ\& usg=AFQjCNFI64jlbWMa6PXyfyJwQjNFuJZ9Ew Consultado, abril 2011.

5. Consejo Nacional de Población (CONAPO). Informe de México: El cambio demográfico, el envejecimiento y la migración internacional en México [Internet]. México: CONAPO, 2008. Disponible en http://www.conapo.gob.mx/prensa/2008/02cepal.pdf Consultado: marzo 2012.

6. Wong R, Espinosa M. Bienestar económico de la población de edad media y avanzada en México. Primeros resultados del Estudio Nacional de Salud y Envejecimiento en México [Internet]. México: ENASEM, 2003. Disponible en http://www.google.com.mx/ url?sa=t\&source=web\&cd=1\&ved=0CBIQFjAA\&url=http\%3A\%2F\%2Fwww.mhas. pop.upenn.edu\%2FPapers\%2Fespanol\%2F7.pdf\&ei=162eTd-WKMja0QGR7tSOB Q\&usg=AFQjCNExHSkz6CFTt2Km8DRSmoQt4rXw_g Consultado: abril 2011.

7. Palloni A, Peláez M. Encuesta sobre salud, bienestar y envejecimiento (SABE) [Internet]. E.U.A.: OMS, 2004 Disponible en http://www.ssc.wisc.edu/sabe/docs/ informeFinal\%20EspaNol\%20noviembre\%202004.pdf Consultado: abril 2011.

8. Ham-Chandé R, González-González C, Orozco-Rocha K, Torres-Martínez A. Análisis y diagnóstico sobre la Encuesta a los Adultos Mayores. México D.F., México: El Colegio de la Frontera Norte; 2005.

9. Sistema Nacional de Información en Salud (SINAIS). Egresos hospitalarios por grupo de edades, según grupo de causas. Tabasco, 2005 [Internet]. México: SINAIS, 2007. Disponible en http://sinais.salud.gob.mx/egresoshospitalarios/ Consultado: abril 2011.

10. Moragas R. Gerontología social, envejecimiento y calidad de vida. $2^{\mathrm{a}}$ ed. Madrid, España: Herder; 1998.

11. Instituto Nacional para el Federalismo y el Desarrollo Municipal. Enciclopedia de los Municipios de México. Tabasco. Historia [Internet]. México: INFDM, 2005 Disponible en http://www.e-local.gob.mx/work/templates/enciclo/tabasco/hist.htm Consultado: abril 2011. 
12. Su B, Shen X, Wei Z. Leisure life in later years: Differences between rural and urban elderly residents inChina. Journal of Leisure Research [Internet]. 2006;38(3):381-97. Disponible en http://www.cerium.ca/IMG/pdf/Baoren_al_2006.pdf Consultado: abril 2011.

13. Alcolea I, Martori E, Sánchez C, Rodríguez-Ávila N. Análisis del tiempo en personas mayores: Organizaciones y actividades en Cataluña (España). Horizonte Sanitario [Internet]. 2003;2(1):25-33. Disponible en http://www.publicaciones.ujat.mx/publicaciones/ horizonte sanitario/ediciones/2003 ene abr/hsv2n1.pdf Consultado: abril 2011.

14. Marín-Sánchez M, García-González AJ, Troyano-Rodríguez Y. Modelo de ocio activo en las personas mayores: Revisión desde una perspectiva psicosocial. SOCIOTAM [Internet]. 2006;XVI(1):147-67. Disponible en http://www.google.commxurl?sa=t\&so urce=web\&cd=1\&ved=0CBIQFjAA\&url=http $\% 3 A \% 2 F \% 2 F r e d a l y c a e m e x F p d f \% 2 F 6$ 54\%2F65416106i=jLeeTbvdCKuC0QGmwvyVBQ\&usg=AFQjCNFYK9Y2Hn3n5jOv jzjHz5CyfeDpZQ Consultado: abril 2011.

15. Sánchez-García M, Gutiérrez-Herrera RF, Gómez-Gómez C. El tiempo libre en los adultos mayores de un área suburbana del Estado de Nuevo León. RESPYN [Internet]. 2005;(Edición Especial 7). Disponible en http://www.respyn.uanl.mx/especiales/2005/ ee-07-2005/documentos/gerontologia.htm Consultado: abril 2011. 